\title{
Signature of Spin Collective Mode in Local Tunneling Spectra of a d-wave Superconductor
}

\author{
Jung Hoon Han \\ Department of Physics, Konkuk University \\ 1 Hwayang-dong, Kwangjin-gu, Seoul 143-701, Korea
}

\begin{abstract}
We consider the influence of magnetic excitations on the local density of states in the $d$-wave superconductor. The magnetic susceptibility is calculated within the renormalized $t-t^{\prime}-J$ model and its influence on the quasiparticle self-energy is considered using a minimal model originally proposed by Polkovnikov et al. cond-mat/0203176. We find the local density of states possess periodic components both along $(\pi, 0)$ and $(\pi, \pi)$ directions with the associated wavevectors changing in magnitude as the quasiparticle energy is varied. Comparison with the STM experiment reveals that the calculated LDOS modulation is inconsistent with the measured data.
\end{abstract}

Introduction: Observation of discernible "checkerboard" patterns in the local density of states (LDOS) has been reported for a superconducting $\mathrm{Bi}_{2} \mathrm{Sr}_{2} \mathrm{CaCu}_{2} \mathrm{O}_{8+\delta}$ (BSCCO) compound in the mixed state, with the spatial periodicity close to four lattice constants 11. Howald et al. reported the same periodicity exists for the LDOS in the same compound but without the magnetic field [2]. Hoffmann et al. in turn argued that this periodicity is in fact a function of energy, i.e. as the probing bias of the scanning tunnelling microscope (STM) is varied the spatial modulation periodicity also changes systematically [3].

A number of theories have emerged in response to such striking set of observations 沺 [6]. Some of these theories emphasize the possible emergence of a new order parameter near the vortex core [4] but, in view of the latest experimental finding [3] may have difficulty to explain how an induced order parameter can exhibit a length scale that depends smoothly on energy. An alternative explanation advanced by Wang and Lee (WL) ascribes the periodicity to the quasiparticle scattering due to localized impurities [5]. In this approach scattering processes connect parts of the underlying Fermi contours at different wavevectors as the quasiparticle energy is varied.

Quite distinct from the proposal of WL is the theory of Polkovnikov, Vojta, and Sachdev(PVS) [6] who showed in a simple model that a dynamic spin fluctuation mode with a distinct ordering wavevector $\mathbf{Q}$ may lead to observable periodic modulations in the local STM spectra at twice $\mathbf{Q}$. They also invoke the local impurity pinning, which is necessary to break the translational symmetry and give rise to spatially varying LDOS. In principle the mechanism proposed by PVS does not depend on the presence of an external magnetic field.

Dynamical spin fluctuations are observable in a number of families of cuprates. It has been shown that magnetic absorption peaks occur at incommensurate wavevectors $(\pi, \pi \pm \delta)$, and $(\pi \pm \delta, \pi)$ with $\delta$ varying with the absorption energy [7]. Brinckmann and Lee (BL) [8] argued that the underlying Fermi surface topology in the superconducting state is responsible for such incommensurate response. Within the Gutzwiller-renormalized
$t-t^{\prime}-J$ model they indeed found the absorption peaks at $(\pi \pm \delta, \pi)$, and $(\pi, \pi \pm \delta)$ while at even lower energies, the incommensurate peaks appear in the diagonal direction, $\left(\pi \pm \delta^{\prime}, \pi \pm \delta^{\prime}\right)$.

Coupled with the idea of PVS, it is worthwhile to ask whether such incommensurate magnetic spectrum will also modulate the LDOS at wavevectors that vary with the energy. In this paper, we examine this issue using the magnetic spectrum calculated from the renormalized $t-t^{\prime}-J$ model. Assuming a simple interaction scheme between the spin of the quasiparticles and the magnetic excitation we evaluate the quasiparticle self-energy and thence the spatially varying LDOS. Ultimately our calculation is aimed at a comparison with the latest experimental data [3], and in the process hope to figure out to what extent, if at all, the magnetic scenario for the observed periodicity is viable.

Methods: We adopt the renormalized $t-t^{\prime}-J$ model [8] as the starting point:

$$
\begin{gathered}
H=-t_{\text {eff }} \sum_{i j} f_{j \sigma}^{\dagger} f_{i \sigma}+t_{\text {eff }}^{\prime} \sum_{i j} f_{j \sigma}^{\dagger} f_{i \sigma}-\mu \sum_{i} f_{i \sigma}^{\dagger} f_{i \sigma} \\
-\frac{J}{4} \sum_{i j}\left(\Delta_{i j}^{*} \epsilon_{\alpha \beta} f_{i \alpha} f_{j \beta}+h . c .\right)+\frac{J_{e f f}}{2} \sum_{i j} S_{i} \cdot S_{j} .
\end{gathered}
$$

In the above, $t_{\text {eff }}=t x+(J / 4) \sum_{\sigma}\left\langle f_{j \sigma}^{\dagger} f_{i \sigma}\right\rangle, t_{e f f}^{\prime}=$ $t^{\prime} x, \Delta_{i j}=\left\langle\epsilon_{\alpha \beta} f_{i \alpha} f_{j \beta}\right\rangle$, and $x$ is the average doping. $J_{\text {eff }}$ is the residual spin-spin interaction between quasiparticles.

From the above mean-field Hamiltonian one calculates the zero-temperature magnetic susceptibility $\chi_{0}(\mathbf{q}, i \nu)$, and through the residual spin-spin interaction $J_{\text {eff }} S_{i} \cdot S_{j}$, the renormalized spin susceptibility

$$
\chi(\mathbf{q}, i \nu)=\frac{\chi_{0}(\mathbf{q}, i \nu)}{1+J(\mathbf{q}) \chi_{0}(\mathbf{q}, i \nu)},
$$

for $J(\mathbf{q})=J_{\text {eff }}\left(\cos q_{x}+\cos q_{y}\right)$. In PVS's treatment the magnetic susceptibility is approximated by a phenomenological form, $\chi_{P V S}(\mathbf{q}, i \nu)^{-1} \propto \nu^{2}+\Delta_{s}^{2}+c^{2}(\mathbf{q}-\mathbf{Q})^{2}$, where $\Delta_{s}$ is the spin gap, and $\mathbf{Q}$ is the ordering wavevector. 
On the other hand, the $t-t^{\prime}-J$ model result for the renormalized magnetic susceptibility is known to exhibit a continuous set of ordering wavevectors with respect to energy 8]. We show a horizontal scan of the imaginary part $\chi^{\prime \prime}(\mathbf{q}, \nu)$ with $q_{y}=\pi$ for a series of energies $\omega$ in Fig. 1. The incommensurate peaks near $q_{x}=\pi$ are obvious, and both these peaks and the less pronounced peaks near $q_{x}=0$ move toward $q_{x}=\pi$ when the energy is raised. In particular the peaks at $(\pi \pm \delta(E), \pi)$ may give rise to a modulation in the charge sector with twice the wavevector [6.9], i.e. at $(2 \pi \pm 2 \delta(E), 2 \pi) \equiv( \pm 2 \delta(E), 0)$. With $\delta(E)$ being a decreasing function of energy, one naively expects LDOS modulation wavevectors to decrease at a higher bias as well, in qualitative agreement with the experiment [3]. Below, we examine through explicit calculation whether this naive argument will hold.

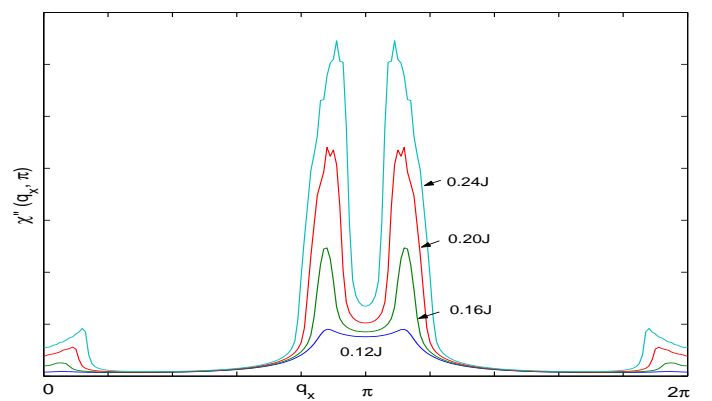

FIG. 1. Imaginary part of the magnetic susceptibility, $\chi^{\prime \prime}(\mathbf{q}, \nu)$ for $q_{y}=\pi$, and $0<q_{x}<2 \pi$ calculated from the renormalized $t-t^{\prime}-J$ model with $J_{\text {eff }}=0.34 J$. Different plots correspond to $\nu=0.12 \mathrm{~J}, 0.16 \mathrm{~J}, 0.20 \mathrm{~J}$, and $0.24 \mathrm{~J}$.

The treatment can proceed by positing the effective action $\mathcal{L}=\mathcal{L}_{M}+\mathcal{L}_{q p}+\mathcal{L}_{q p-M}$ consisting respectively of the magnetic and quasiparticle sectors and their coupling. For the magnetic part [6],

$$
\begin{aligned}
\mathcal{L}_{M}\left[\Phi, \Phi^{*}\right] & =\frac{1}{2} \int d^{3} x d^{3} y \Phi^{*}(x) \chi^{-1}(x-y) \Phi(y) \\
& -\frac{1}{2} \int d \tau\left[\xi^{*} \Phi^{2}\left(\mathbf{r}_{i}, \tau\right)+\xi \Phi^{* 2}\left(\mathbf{r}_{i}, \tau\right)\right]
\end{aligned}
$$

Space-time coordinates are denoted by $x=(\mathbf{r}, \tau)$ and $y=\left(\mathbf{r}^{\prime}, \tau^{\prime}\right)$. The last term is the pinning potential, located around $\mathbf{r}_{i}$, for the spin density wave(SDW). Due to the pinning term the propagator $\left\langle\Phi^{*}(\mathbf{r}, i \omega) \Phi\left(\mathbf{r}^{\prime}, i \omega\right)\right\rangle \equiv$ $\chi\left(\mathbf{r}, \mathbf{r}^{\prime}, i \omega\right)$ is no longer translationally invariant.

The quasiparticle coupling to the fluctuating SDW is modelled by [6]

$$
\mathcal{L}_{q p-M}=\frac{1}{2} g S(\mathbf{r} \tau) \cdot\left[\Phi(\mathbf{r} \tau)+\Phi^{*}(\mathbf{r} \tau)\right]
$$

Through this term the quasiparticle propagator is influenced by the collective mode, and exhibits translational symmetry breaking. The perturbative treatment of the effective interaction is straightforward, with the one-loop result for the change in Green's function $\delta \mathcal{G}$ [10,

$$
\begin{gathered}
\delta \mathcal{G}\left(\mathbf{r}, \mathbf{r}^{\prime}, i \omega\right)=\sum_{\mathbf{r}_{1}, \mathbf{r}_{2}, i \nu} g^{2}(i \nu) \chi\left(\mathbf{r}_{1}-\mathbf{r}_{i}, i \nu\right) T(i \nu) \chi\left(\mathbf{r}_{i}-\mathbf{r}_{2}, i \nu\right) \\
\times G\left(\mathbf{r}-\mathbf{r}_{1}, i \omega\right) G\left(\mathbf{r}_{1}-\mathbf{r}_{2}, i \omega-i \nu\right) G\left(\mathbf{r}_{2}-\mathbf{r}^{\prime}, i \omega\right)
\end{gathered}
$$

where $T(i \nu)=\chi(\mathbf{r}=0, i \nu)$. Frequency dependence in the coupling $g(i \nu)$ is introduced for generality. In arriving at this expression we have only kept those terms that break the translational symmetry and ignored the space-independent self-energy correction. After the Wick rotation, $i \omega \rightarrow \omega+i \delta$, the spatially varying LDOS is $N(\mathbf{r}, \omega)=(1 / \pi) \operatorname{Im} \delta \mathcal{G}(\mathbf{r}, \mathbf{r}, \omega+i \delta)$.

Normally the summation $\sum_{i \nu}$ is carried out analytically, and the resulting expression is evaluated by numerical means after the Wick rotation $i \omega \rightarrow \omega+i \delta$. In our case the renormalized susceptibility $\chi(\mathbf{q}, i \nu)$ does not possess a simple Lehmann expansion, which prevents the frequency summation from being carried out. Instead we resort to the time-ordered Green's function method (as opposed to the Matsubara Green's function method) which does not require the Wick rotation. At zero temperature LDOS can be obtained from the time-ordered Green's function by ( $T=$ time-ordered)

$$
N(\mathbf{r}, \omega)=(1 / \pi) \operatorname{sgn}(\omega) \operatorname{Im} \delta \mathcal{G}_{T}(\mathbf{r}, \mathbf{r}, \omega)
$$

Perturbation theory proceeds in entirely analogous manner, and we only quote the final result for the Green's function correction.

$$
\begin{aligned}
& \delta \mathcal{G}_{T}\left(\mathbf{r}, \mathbf{r}^{\prime}, \omega\right)=-i \sum_{\mathbf{r}_{1}, \mathbf{r}_{2}} \int_{\nu} g^{2}(\nu) \chi_{T}\left(\mathbf{r}_{1}-\mathbf{r}_{i}, \nu\right) T(\nu) \chi_{T}\left(\mathbf{r}_{i}-\mathbf{r}_{2}, \nu\right) \\
& \quad \times G_{T}\left(\mathbf{r}-\mathbf{r}_{1}, \omega\right) G_{T}\left(\mathbf{r}_{1}-\mathbf{r}_{2}, \omega-\nu\right) G_{T}\left(\mathbf{r}_{2}-\mathbf{r}^{\prime}, \omega\right)
\end{aligned}
$$

The time-ordered susceptibility $\chi_{T}$ and Green's function $G_{T}$ are obtained from their Matsubara counterparts by $i \omega \rightarrow \omega+i \operatorname{sgn}(\omega)$

Results: We evaluate Eq. (7) numerically on a large lattice of dimension $[201 \times 201]$ using self-consistently determined parameters $\Delta_{\max } \equiv 2\left|\left\langle\epsilon_{\alpha \beta} f_{i \alpha} f_{j \beta}\right\rangle\right|=0.48 J$. The impurity site is located at the center, $\mathbf{r}_{i}=(101,101)$. We use $J_{\text {eff }}=0.34 \mathrm{~J}$, and the broadening factor $\delta=0.01 J$ in evaluating $\chi(\mathbf{r}, \nu)$ and $G(\mathbf{r}, \omega)$. The $\nu$ integration is replaced with a discrete sum, $\int d \nu f(\nu) \rightarrow$ $\Delta \nu \sum_{s} f(s \Delta \nu)$, where $s$ is an integer, $-s_{\max } \leq s \leq s_{\max }$. The following results are taken with $\Delta \nu=0.04 \mathrm{~J}$, and $s_{\max }=14$ implying that virtual processes with the offshell energy of up to $0.56 \mathrm{~J}$ are taken into account in the self-energy evaluation. The commensurate response at $\mathbf{q}=(\pi, \pi)$ in the susceptibility occurs at $\nu_{\text {res }} \approx 0.32 J$ for the present model, while at energies above and below $\nu_{r e s}$, the resonance occurs away from $(\pi, \pi)$ [8]. Our selfenergy energy calculation therefore includes effects from both above and below the resonance energy. We have confirmed on a smaller lattice that using a finer scale 
$\Delta \nu=0.02 J$ does not change the final result qualitatively. The on-site Green's function $\delta \mathcal{G}_{T}(\mathbf{r}, \mathbf{r}, \omega)$ is evaluated for a subset of the lattice, typically for $\mathbf{r}$ lying in $[101 \times 101]$ about the impurity center, for $|\omega|$ up to $0.32 J$. The realspace LDOS thus obtained is then Fourier-transformed according to $N(\mathbf{q}, \omega)=\left|\sum_{\mathbf{r}} e^{-i \mathbf{q} \cdot \mathbf{r}} N(\mathbf{r}, \omega)\right|$.

FIG. 2. Fourier-transformed LDOS $N(\mathbf{q}, \omega)$ for the $\mathbf{q}$-range $[-\pi, \pi] \times[\pi, \pi]$ and for $-0.32 J \leq \omega \leq 0.32 J$. Center of each rectangle corresponds to $\mathbf{q}=(0,0)$. The intensity scheme is with respect to each figure, and should not be compared between different plots. (Please refer to attached figure ldosk100b-v2.jpg)

Plots in Fig. 2 show $N(\mathbf{q}, \omega)$ calculated with the coupling constant $g^{2}(\nu)=$ const. Apart from the $\mathbf{q}=0$ peak, we find some prominent peaks along both diagonal (meaning $( \pm \pi, \pm \pi))$ and horizontal (meaning $( \pm \pi, 0)$ and $(0, \pm \pi))$ directions. The peaks are located near the ends of the Brillouin zone at a small bias, and move toward $\mathbf{q}=0$ as the bias energy is increased. A horizontal(diagonal) scan of $N(\mathbf{q}, \omega)$ allows the identification of wavevectors $\mathbf{q}_{h}(\omega)\left(\mathbf{q}_{d}(\omega)\right)$ for which $N(\mathbf{q}, \omega)$ is a local maximum. The values of $q_{d}(\omega) \equiv\left|\mathbf{q}_{d}(\omega)\right| / \sqrt{2}$ and $q_{h}(\omega) \equiv\left|\mathbf{q}_{h}(\omega)\right|$ are plotted in Fig. $4(\mathrm{a})$. Clearly both $q_{d}(\omega)$ and $q_{h}(\omega)$ are decreasing functions of energy. The detailed shape of $N(\mathbf{q}, \omega)$ however depends somewhat on whether $V$ is negative (electrons tunneling out) or positive (electrons tunneling in).

As shown in Fig. 1 the magnetic absorption peaks are most pronounced around $(\pi, \pi)$. Note however that the Green's function shown in Eqs. (5) and (7) are a product of several complicated functions, and the magnetic absorption spectrum alone does not a priori characterize the LDOS. In this regard we can loosely classify the structures in $\chi^{\prime \prime}(\mathbf{q}, \nu)$ as those near $(\pi, 0),(0, \pi)$, and those near $(\pi, \pi)$. In the result shown in Fig. 2 contributions from these two regions will undoubtedly be mixed. Instead, if we truncate the magnetic spectrum to be within a certain vicinity of $(\pi, \pi)$, the quasiparticle Green's function will also be influenced by the magnetic excitations at these wavevectors, and none from around $(\pi, 0),(0, \pi)$. This is achieved by using the reduced susceptibility $\chi_{\text {red }}(\mathbf{q}, \nu)=\chi(\mathbf{q}, \nu)$ for $-\pi / 4<q_{x}, q_{y}<\pi / 4$, $\chi_{\text {red }}(\mathbf{q}, \nu)=0$ otherwise. Its Fourier transform $\chi_{\text {red }}(\mathbf{r}, \nu)$ is used in the evaluation of the new LDOS, $N_{\text {red }}(\mathbf{r}, \omega)$ and $N_{\text {red }}(\mathbf{q}, \omega)$, shown in Fig. 3. In this calculation we use the cutoff $g^{2}(\nu)=g^{2}(0) \exp \left[-0.1(\nu / \Delta \nu)^{2}\right]$. For a less sharp cutoff such as $g^{2}(\nu)=g^{2}(0)$ or $g^{2}(\nu)=$ $g^{2}(0) \exp [-0.1|\nu / \Delta \nu|]$, we find no other distinguishable structure in $N_{\text {red }}(\mathbf{q}, \omega)$ except a broad hump centered at $\mathbf{q}=0$.
FIG. 3. LDOS $N_{\text {red }}(\mathbf{q}, \omega)$ calculated with the reduced magnetic susceptibility $\chi_{\text {red }}$. Energy and momentum ranges are the same as in Fig. 2 (Please refer to attached figure ldosk200e.jpg)

There is very little overlap between the two figures 2 and 3, as also reflected in the $q_{d}(\omega)$ and $q_{h}(\omega)$ values obtained respectively from each figure. Figure $1(\mathrm{~b})$ shows the peak intensity position of $N_{\text {red }}(\mathbf{q}, \omega)$. Essentially all $q_{d}(\omega), q_{h}(\omega)$ are greater than $\pi / 2$ in the $N(\mathbf{q}, \omega)$ while in the reduced LDOS, all $q_{d}(\omega)$, and $q_{h}(\omega)$ are less than $\pi / 2$. Furthermore, $q_{d}(\omega)$ and $q_{h}(\omega)$ in Fig. 1(b) are increasing functions of energy, as opposed to Fig. 团(a) where they are decreasing. In both plots, $q_{d}(\omega)$ and $q_{h}(\omega)$ show the same dependence on energy, being both increasing or both decreasing functions. As previously mentioned a standard Ginzburg-Landau argument about the coupling between SDW and charge-densitywave (CDW) 9] implies that an ordering tendency occurring at $(\pi \pm \delta, \pi \pm \delta)$ for the SDW will lead to CDW modulations at $( \pm 2 \delta, \pm 2 \delta)$. Naively, since $\delta$ decreases with energy, one expects $q_{h}(\omega)$ in Fig. 1 (b) to be a decreasing function of $\omega$, which is contradicted by our calculation. In obtaining Fig. 3 we (1) truncated the magnetic spectrum within a small momentum window around $(\pi, \pi)$ and (2) modified the coupling $g^{2}(\nu)$. With this manipulation we were able to bring out features in the LDOS which were "hidden" in the calculation that led to Fig. 2. Since the coupling constant $g$ must in reality be a function of both momentum and energy, one cannot be too certain a priori which of the features found in Figs. 2 and 3 are more readily observable in an experiment.
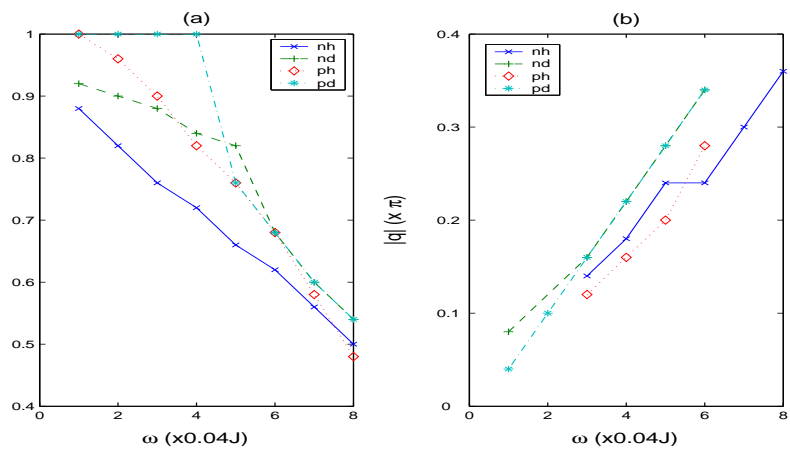

FIG. 4. Energy dependence of $q_{d}(\omega)$ and $q_{h}(\omega)$ (defined in the text) obtained from $N(\mathbf{q}, \omega)$ (Figure (a)) and from $N_{\text {red }}(\mathbf{q}, \omega)$ (figure (b)). In each figure, four data sets are shown for $q_{d}(\omega<0)$ (nd), $q_{h}(\omega<0)(\mathrm{nh}), q_{d}(\omega>0)(\mathrm{pd})$, and $q_{h}(\omega>0)(\mathrm{ph})$ against $|\omega|$. Note that all the $q$ values decrease with energy $|\omega|$ in (a), and increase in (b).

Discussion: We now come to comparison of our result with the experimental data. First, it has been found in Ref. [3] that while $q_{d}(\omega)$ increases, $q_{h}(\omega)$ decreases at a larger bias $|\omega|$. This is in contrast to our calculation where both $q_{d}(\omega)$ and $q_{d}(\omega)$ behave analogously with respect to energy. Secondly, experimentally mea- 
sured $k_{h}(\omega)$ values are found to lie below $\pi / 2$ for a wide range of energies and hole doping, which may be consistent with Figure $4(\mathrm{~b})$, but in this case the energy dependence is opposite to our calculation. In Fig. 4(a), $q_{d}(\omega)$ and $q_{h}(\omega)$ values are rather high which, translated into real space, implies LDOS modulations on the length of 2-4 lattice spacings. While such magnetic-fluctuationinduced LDOS variations are not forbidden and may very well be observable in the future, we must conclude that the LDOS modulation based on the $t-t^{\prime}-J$ model of the magnetic excitation is inconsistent with the currently known experiment [3].

It is possible that the renormalized $t-t^{\prime}-J$ model does not after all capture the quasiparticle band structure of the BSCCO and that in another model, one indeed finds the energy dependence of $q_{d}$ and $q_{h}$ consistent with the experiment within the magnetic fluctuation scenario. For example, in WL [5], the model adopted is the phenomenological tight-binding model originally proposed by Norman [1]].

On the theoretical side, one should be careful that the naive Ginzburg-Landau argument for SDW-CDW coupling may in some cases lead to predictions which are inconsistent with a full many-body calculation such as this one. Although the patterns in $N(\mathbf{q}, \omega)$ are ultimately due to the underlying magnetic fluctuation, the reason for the particular energy dependence of the modulation period found in Figs. 2 and 3 remains unclear. Finally an entirely different mechanism such as WL's are not incompatible with the present model, and may well simultaneously lead to observable effects in a given system.

We thank Seamus Davis, Dung-Hai Lee, and QiangHua Wang for reading the manuscript and for critical comments. We acknowledge the financial support from the Faculty Fund of Konkuk University. This work is also supported in part by the Korean Science and Engineering Foundation (KOSEF) through the Center for Strongly Correlated Materials Research (CSCMR).

[1] J. E. Hoffman et al., Science 295, 466 (2002).

[2] C. Howald, H. Eisaki, N. Kaneko, and A. Kapitulnik, cod-mat/0201546.

[3] J. E. Hoffman, K. McElroy, D.-H. Lee, K. M. Lang, H. Eisaki, S. Uchida, and J. C. Davis, To appear in Science.

[4] Jian-Xin Zhu, Ivar Martin, and A. R. Bishop, condmat/0201519: M. Franz, D. E. Sheehy, and Z. Tesanovic, cond-mat/0203219; Han-Dong Chen et al., condmat/0203332: Daniel Podolsky et al., cond-mat/0204011; M. Vojta, cond-mat/0204284.

[5] Qiang-Hua Wang and Dung-Hai Lee, cond-mat/0205118.

[6] Anatoli Polkovnikov, Matthias Vojta, and Subir Sachdev, cond-mat/0203176.

[7] H. Mook, P. Dai, S. M. Hayden, G. Aeppli, T. G. Perring, and F. Dogan, Nature (London) 395, 580 (1998); P. Bourges, Y. Sidis, H. F. Fong, L. P. Regnault, J. Bossy, A. Ivanov, and B. Keimer, Science 288, 1234 (2000).

[8] Jan Brinckmann and Patrick A. Lee, Phys. Rev. Lett. 82, 2915 (1999); Jan Brinckmann and Patrick A. Lee, Phys. Rev. B 65, 014502 (2001).

[9] Oron Zachar, S. A. Kivelson and V. J. Emery, Phys. Rev. B 57, 1422 (1998).

[10] Actual result involves a multiplicative factor of order unity, which we absorb into the definition of the coupling $g$.

[11] M. R. Norman, Phys. Rev. B 61, 14751 (2000); M. R. Norman, ibid. 63, 092509 (2001). 
This figure "ldosk100b-v2.jpg" is available in "jpg" format from: http://arxiv.org/ps/cond-mat/0206284v1 
This figure "ldosk200e.jpg" is available in "jpg" format from: http://arxiv.org/ps/cond-mat/0206284v1 